\title{
Microscopie non linéaire
}

\author{
L. Canioni, R. Barillé, A. Brocus et L. Sarger
}

CPMOH, Université de Bordeaux l, 351 cours de la Libération, 33405 Talence cedex, France

\begin{abstract}
Résumé :La microscopie non linéaire par mélange à quatre ondes permet l'obtention d'images sans traitement particulier des échantillons souvent difficile à mettre en cuvre sur des échantillons biologiques mais impossible à réaliser sur des échantillons massifs [1]. La validité de cette technique a été démontrée dans le cas particulier de la microscopie par génération de tierce harmonique sur des échantillons biologiques [2]. Les mécanismes d'accord de phase qui conditionnent le contraste et la résolution attendue pour cette microscopie cohérente non linéaire par mélange à quatre ondes permettent de donner une limite à ce type d'imagerie. Nous présentons dans cet article quelques exemples d'applications qualitatives et quantitatives de cette imagerie.
\end{abstract}

\section{MICROSCOPIE COHERENTE NON LINEAIRE}

Parallèlement au progrès de la chimie et du génie génétique qui permettent une microscopie fonctionnelle et quantitative des tissus et cellules vivantes, des problèmes restent ouverts dans lesquels une microscopie cohérente non linéaire peut apporter des solutions. En effet, les problèmes d'imagerie à travers des milieux diffusants que constitue quelque $100 \mu \mathrm{m}$ de tissus vivants mais également la difficulté de charger avec un fluorophore ces tissus sont autant de difficultés que la microscopie non linéaire peut circonscrire. La toxicité des substances chimiques qui servent d'agent de contraste et la diffusion multiple de la lumière rendent parfois l'imagerie confocale inopérante. La microscopie cohérente par mélange d'onde du fait de son caractère non linéaire de l'excitation permet, comme la microscopie à deux photons, une imagerie localisée au voxel représcnté par la tache focale de l'onde laser focalisée. Par ailleurs en utilisant comme agent de contraste les propriétés physiques intrinsèques du matériau, la susceptibilité non linéaire, il est maintenant possible de réaliser des images en microscopie sans préparation particulière des échantillons.

\section{1 agent de contraste en microscopie non linéaire}

La polarisation non linéaire est donc le mécanisme de contraste de cette microscopie. Le premier terme de cette polarisation toujours présent dans tous les matériaux indépendamment de lour symétrie est celui du troisième ordre $\chi^{(3)}$. Celui-ci mélange trois champs excitateurs par le biais de la susceptibilité non linéaire pour générer une polarisation qui oscille à la fréquence composite des champs excitateurs.

$$
\mathbf{P}(\omega)=\chi \cdot \mathbf{E}+\chi^{(2)} \cdot \mathbf{E}^{2}+\chi^{(3)} \cdot \mathbf{E}^{3}+\ldots \cdot
$$

Des mesures de susceptibilité non linéaire ont montré que même sur des matériaux transparents de même indice de réfraction (la silice fondue) de faibles impuretés conduisaient à plus de $30 \%$ de variation de la susceptibilité non linéaire $\chi^{(3)}$. Ces grandes différences permettent une exaltation du contraste qu'il faudra sans doute intégrer comme une nouvelle dimension dans l'interprétation des images. Du fait de la forte focalisation, le régime non linéaire de l'interaction est facilement atteinte avec un laser impulsionnel femtoseconde délivrant de faibles puissances moyennes compatibles avec les applications en science du vivant.

Nous allons aborder maintenant dans le cas concret de la microscopie de tierce harmonique comment la polarisation non linéaire en tant qu'agent de contraste nous permet d'obtenir l'image d'un objet. 


\section{MICROSCOPIE PAR GENERATION DE TIERCE HARMONIQUE (GTH)}

Au point focal du faisceau laser intense excitateur à la longueur d'onde $\lambda$, la polarisation non linéaire est un terme source pour une onde cohérente en transmission correspondant à la troisième harmonique, $\lambda / 3$ pour connaître l'amplitude du champ harmonique, l'accord de phase en faisceau colinéaire $\Delta k=k(3 w)-3 k(w)$, avec $k$ vecteur d 'onde est généralement suffisant. Dans les matériaux usuels $\Delta k<0$, ce qui est défavorable pour la détection d'un signal harmonique.

Il faut pour être plus précis, tenir compte de la forte focalisation de notre faisceau laser pour donner une notion d'accord de phase en faisceau focalisé. Ecrivons pour cela, l'équation de propagation de l'onde harmonique dans le cas de l'enveloppe lentement variable:

$$
2 \mathrm{ik}_{3 \omega} \frac{\partial \mathrm{A}_{3 \omega}}{\partial \mathrm{z}}+\frac{\partial^{2} \mathrm{~A}_{3 \omega}}{\partial \mathrm{x}}+\frac{\partial^{2} \mathrm{~A}_{3 \omega}}{\partial \mathrm{y}}=-\frac{4 \pi \omega^{2}{ }^{3 \omega}}{\mathrm{c}^{2}} \chi^{(3)} \mathrm{A}_{\omega}^{3} \exp (\mathrm{i} \Delta \mathrm{kz})
$$

Cette équation de propagation paraxiale est une approximation qui peut être utilisée pour de faibles focalisations $(\mathrm{NA}<0.7)$ elle admet néanmoins une solution analytique approchée $\mathrm{si}|\Delta \mathrm{k}|<5 \mu \mathrm{m}-1$ qui permet dès à présent une discussion des régimes :

$$
A_{3 \omega}(z)=\frac{i 2 \pi 3 \omega}{n c} \chi_{3} A_{\omega}^{3} J_{3 \omega}\left(\Delta k, z_{0}, z\right)
$$

La troisième harmonique émise suit le profil d'une onde gaussienne cohérente de beam waist diminué d'un facteur $\sqrt{3}$ par rapport au champ fondamental. L'amplitude de l'onde harmonique dépend de deux termes : l'amplitude de la susceptibilité non linéaire et le facteur $\mathbf{J}$ que l'on peut associer physiquement à la longueur d'interaction effective en troisième harmonique (TH) :

$$
J_{3 \omega}\left(\Delta k, z_{o}, z\right)=\int_{z_{o}}^{z} \frac{e^{i \Delta k z^{\prime}} d z^{\prime}}{\left(1+2 i z^{1} / b\right)^{2}}
$$

$\mathrm{b}$ est la longueur de Rayleigh. On remarque que $\mathrm{J}=0$ pour des matériaux massifs. En effet, la longueur de l'échantillon est beaucoup plus grande que b et il n'y a pas de TH mesurable dans ces échantillons. Ceci traduit le caractère cohérent de l'émission qui se construit par interférences constructives de part et d'autre du beam waist mais qui in fine donne un signal nul car le champ de par et d'autre du beam waist présente un saut de phase de $\mathrm{Pi}$ et donc un taux d'interférences complètement destructives. Que se passe t'il si le matériau présente une interface?

Figure 1 Diagramme d'émission de la $\mathrm{TH}$ avec une interface vide matériaux placée au beam waist et $\Delta k$ négatif. En noir l'intensité excitatrice

Dans le cas d'une interface : le

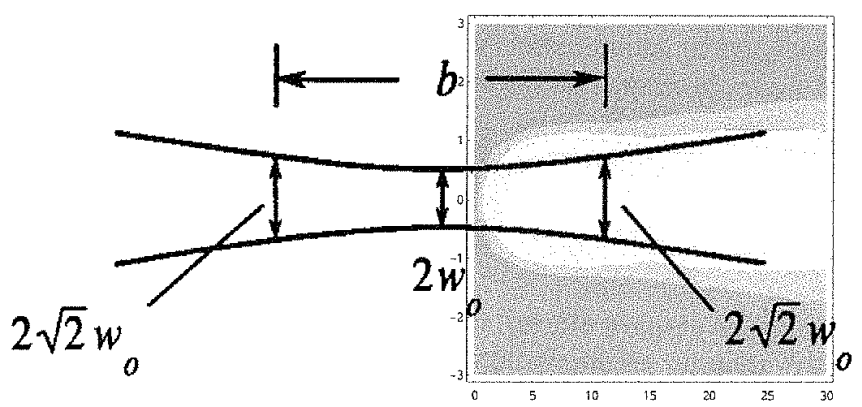
taux d'interférences destructives n'est pas maximum de par et d'autre du beam waist et il reste un champ harmonique qui se propage en dehors de la zone d'interaction. Le contraste dépend de deux paramètres : la différence de $\chi^{(3)}$ et du $D(\Delta k)$ entre les deux milieux qui conduit à une GTH non nulle. Le signal TH varie comme $1 / \mathrm{D}(\Delta \mathrm{k})^{2}$ et comme $D\left(\left|\chi^{(3)}\right| 2\right)[3]$. 


\subsection{Expérience}

Le microscope est décrit dans la figure 2. Les objectifs utilisés ont une ouverture numérique de 1.3 et 0.7 la puissance laser moyenne est de $2 \mathrm{~mW}$ sur l'échantillon. En vertu de l'équation 3 la résolution attendue est de $1.5 \mu \mathrm{m}$ en $z$ et $0.5 \mu \mathrm{m}$ en latéral. L'intensité du signal G'TH est d'environ de 3 photons par pulse laser excitateur

Figure 2 Dispositif expérimental avec la source laser OPO synchrone OPAL de Spectra Physics @1.4-1.6 $\mu \mathrm{m} 80$ $\mathrm{MHz}, 350 \mathrm{~mW}$ Filtrage spatial et scanner. La TH@ 0.5 $\mu \mathrm{m}$ environ est filtrée par un filtre BG11 de Melles Griot et enregistrée par un photo multiplicateur Hamamatsu H7732-01

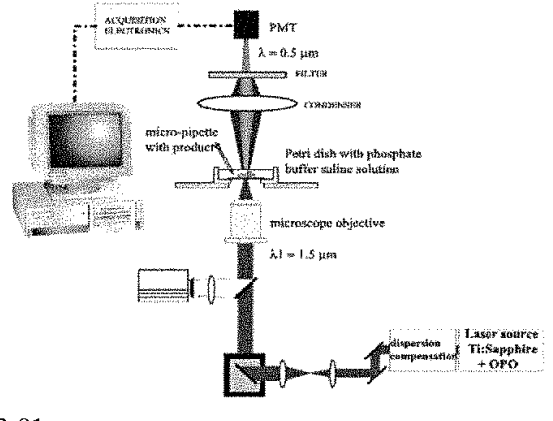

\subsection{Applications}

Susceptibilité non linéaire de couches minces: Expérience

La première application potentielle de cette microscopie concerne la mesure de la susceptibilité non linéaire de couches minces.

Figure 3 : le liquide qui représente la couche mince est placé entre une lamelle de microscope d'épaisseur $100 \mu \mathrm{m}$ et d'une lame de verre. Le col du faisceau est déplacé du haut vers le bas. Deux interfaces sont sondées air silice et silice liquide

Le signal est proportionnel à la différence de $\chi^{(3)_{2}}$ entre les deux

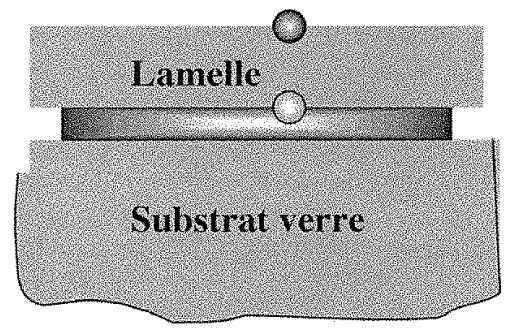
matériaux suivant l'équation (3). La première interface sert de référence pour calibrer le système. La valeur de $b$ est mesurée in situ avec une photodiode à deux photons, le désaccord de phase est mesuré ailleurs entre les différents matériaux. Un exemple des profils verticaux de troisième harmonique est représenté figure 4. La différence de hauteurs entre les deux interfaces dépend de façon non linéaire du désaccord de phase et de la susceptibilité. Pour avoir la meilleure sensibilité, l'ouverture numérique de l'objectif est choisie égale à 0,7
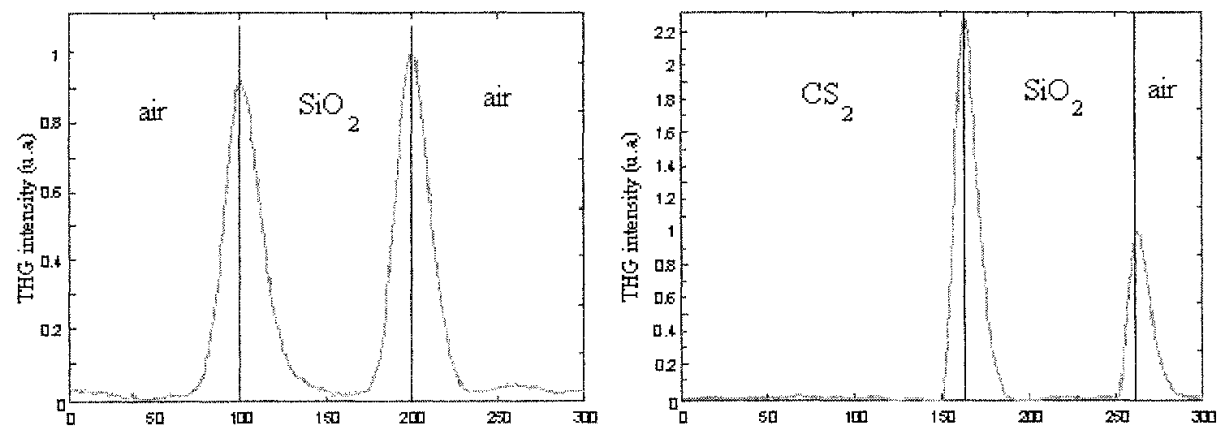

Figure 4. Signal de troisième harmonique à l'interface air verre et l'interface verre air ou verre liquide. Le premier graphique nous permet de vérifier la sensibilité de la méthode (environ $20 \%$ de fluctuation entre le premier et le second pic) 
Les résultats obtenus sont résumés dans le graphique suivant (figure 5) et comparés aux références bibliographiques pour ces différents liquides. Il faut noter que le signal de GrTH n'est originel qu'à la partie électronique de la polarisation.

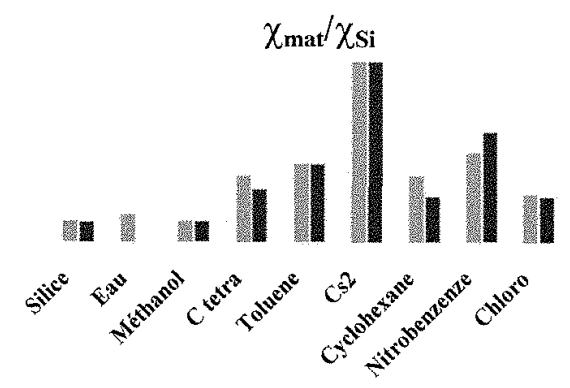

Figure 5. Comparaison des mesures obtenues pour différents liquides- en gris nos résultats en noirs les références.

Détermination de la Structure transverse de fibres optiques de façon non destructive

Une des applications potentielles de la GTH est la détermination des structures internes des objets. Pour les fibres optiques le problème est souvent crucial notamment pour le contrôle qualité de fibre multi cœur comme les fibres de Bragg DCI. Jusqu'à présent seules les méthodes destructrices permettaient de déterminer les paramètres spatiaux de ces objets.

La figure 6 compare le signal de GTH obtenu a différentes hauteurs des fibres suivant un axe. On remarque la différence de signaux pour les deux types de fibres. A droite, la fibre monomode présente un signal monolithique avec deux épaulements $P_{1}, P_{2}$ qui signent la présence du coeur de la fibre. Dans ce cas la lecture du signal de troisième harmonique est immédiate et permet une reconstruction directe de la structure interne de la fibre.

Figure 6. Vue en coupe par GTH d'une fibre monomode et d'une fibre DCI. En insert, la photo d'une fibre $\mathrm{DCl}$ coupée pour comparaison dans cette expérience. Le trait blanc représente une des zones où l'on effectue le balayage du faisceau en

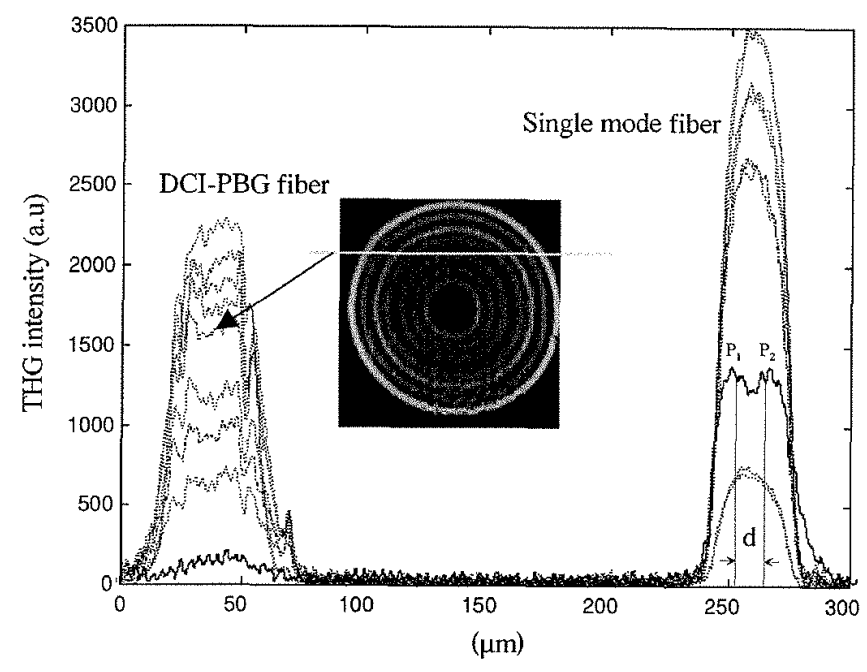
GTH.

Pour la fibre DCI on remarque que le signal est notablement différent et présente de nombreuses oscillations au regard de la complexité du système. On remarque que le signal GTH n'est pas ici homothétique avec l'image de l'objet. Il n'est pas possible d'assigner donc directement la structure. Nous pensons que comme pour les techniques d'ellipsomètrie le caractère cohérent de la formation d'images nécessite une déconvolution. Le résultat sur la structure est donc modèle dépendant. 


\section{Conclusion}

La microscopie cohérente non linéaire permet une visualisation 3D d'objets sans adjonction d'agent de contraste. De nombreuses applications existent tant dans le domaine des sciences des matériaux que dans celles du vivant. Cette microscopie est suffisamment adaptable pour envisager de nombreuses extensions. En effet, tous les paramètres responsables du contraste n'ont pas encore été étudiés : la résolution temporelle, spectrale et le jeu sur la polarisation de l'onde cohérente.

Des progrès sont encore nécessaires pour apprendre à lire ces images cohérentes notamment pour les objets à structuration complexe où des algorithmes restent à développer pour retrouver la forme de l'objet comme le suggère la dernière application présentée sur l'imagerie des fibres.

\section{Références:}

[1] D. Yelin a,d Y. Silberberg, Opt. Exp., 5(8), 169, (1999); A. C. Millard, P. W. Wiseman, D. N. Fittinghoff, K. R. Wilson, J. A. Squier, and M. Müller, Appl. Opt., 38(36), 7393, (1999).

[2] L. Canioni, S. Rivet, L. Sarger, R. Barille, P. Vacher, P. Voisin, Opt. Lett., 26(8), 515-517, (2001).

[3] T. Y. F. Tsang, Phys. Rev. A 52, 4116-4125 (1995). 